\title{
An E3 Ubiquitin Ligase RNF139 Serves as a Tumor-Suppressor in Glioma
}

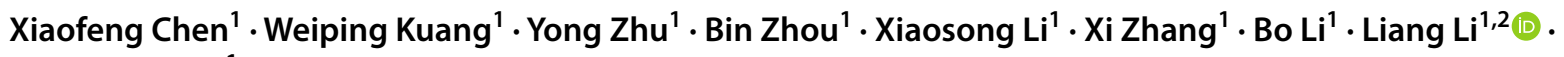 \\ Shucheng Zou ${ }^{1}$
}

Received: 10 March 2021 / Accepted: 14 May 2021 / Published online: 9 June 2021

(c) The Author(s) 2021

\begin{abstract}
Glioma is highly lethal because of its high malignancy. Ubiquitination, a type of ubiquitin-dependent protein modification, has been reported to play an oncogenic or tumor-suppressive role in glioma development, depending on the targets. Ring finger protein 139 (RNF139) is a membrane-bound E3 ubiquitin ligase serving as a tumor suppressor by ubiquitylationdependently suppressing cell growth. Herein, we firstly confirmed the abnormal downregulation of RNF139 in glioma tissues and cell lines. In glioma cells, ectopic RNF139 overexpression could inhibit, whereas RNF139 knockdown could aggravate the aggressive behaviors of glioma cells, including hyperproliferation, migration, and invasion. Moreover, in two glioma cell lines, RNF139 overexpression inhibited, whereas RNF139 knockdown enhanced the phosphorylation of phosphatidylinositol 3-kinase (PI3K) and AKT serine/threonine kinase 1 (AKT). In a word, we demonstrate the aberration in RNF139 expression in glioma tissue samples and cell lines. RNF139 serves as a tumor-suppressor in glioma by inhibiting glioma cell proliferation, migration, and invasion and promoting glioma cell apoptosis through regulating PI3K/AKT signaling.
\end{abstract}

Keywords Glioma $\cdot$ E3 ubiquitin ligase $\cdot$ RNF139 $\cdot$ The PI3K/AKT signaling

\section{Introduction}

Glioma, the most common primary intracranial tumor accounting for more than $80 \%$ of craniocerebral malignant tumors, is highly lethal because of its high malignancy (Ostrom et al. 2014). During the past decades, genomic analysis of gliomas has provided new evidence for risk and prognosis. For example, critical events reported include the methylation of $\mathrm{O}^{6}$-methylguanine-DNA methyltransferase (Chen et al. 2013; Esteller et al. 2000), the mutation in isocitrate dehydrogenase (Brennan et al. 2013), the ubiquitination and degradation of key factors depending on ubiquitin

Liang Li

superliliang@126.com

$\triangle$ Shucheng Zou

2297128086@qq.com

1 Department of Neurosurgery, Hunan Brain Hospital, Clinical Medical School of Hunan, University of Chinese Medicine, Changsha 410007, Hunan Province, China

2 Provincial Key Laboratory of TCM Diagnostics, Hunan University of Chinese Medicine, Changsha 410208, Hunan Province, China
(Ciechanover 2005; Muratani and Tansey 2003; Pan et al. 2015; Sang et al. 2019), and so on. These biomarkers might also be promising treatment targets.

Protein modification by ubiquitin, known as ubiquitination, could regulate proteasomal degradation, thus affecting multiple cellular processes, such as cell cycle regulation (Koepp et al. 1999), cellular response to inflammation (Ghosh et al. 1998), and antigen presentation (Rock and Goldberg 1999). As a result, the aberrations of ubiquitination, not surprisingly, might be associated with multiple pathologic states including cancer. Notably, either oncogene or suppressor gene products could be the targets of ubiquitination (Ciechanover et al. 1991, 1984; Kibel et al. 1995; Rogers et al. 1986); thus, deregulated proteasomal degradation, depending on the targets, could either play an oncogenic or tumor-suppressive role. In general, a three-tiered enzymatic cascade, including E1 (also known as ubiquitin-activating enzyme), E2 (also known as ubiquitin-conjugating enzyme), and E3 (also known as ubiquitin ligases), is necessary for tuning the whole ubiquitination (Pickart 2001; Zheng and Shabek 2017). Since the binding of proper E3 ubiquitin ligase to the target protein is an indispensable step for ubiquitination, E3 ubiquitin ligases are central functional modules during ubiquitination. 
Table 1 ShRNA sequence of RNF139

\begin{tabular}{ll}
\hline Gene & Sequence $\left(5^{\prime}-3^{\prime}\right)$ \\
\hline shRNA1 & \\
$\begin{array}{l}\text { Top strand } \\
\text { Bottom strand } \\
\text { shRNA2 }\end{array}$ & AACCGCCTTTCTGTTAGCTGCAACTCGAAAGTTGCAGCTAACAGAAAGGC \\
Top strand & CACCGCACTTTGCCTTCGGAAATGGCGAACCATTTCCGAAGGCAAAGTGC \\
Bottom strand & AAAAGCACTTTGCCTTCGGAAATGGTTCGCCATTTCCGAAGGCAAAGTGC \\
shRNA-NC & \\
$\begin{array}{l}\text { Top strand } \\
\text { Bottom strand }\end{array}$ & CACCGCCTTTCTGTTAGCTGCAACTCGAAAGTTGCAGCTAACAGAAAGGC \\
\hline
\end{tabular}

RNF139 (also known as TRC8) is a membrane-bound E3 ubiquitin ligase, which plays a tumor-suppressive role (Gemmill et al. 1998) by ubiquitylation-dependently suppressing cell growth (Brauweiler et al. 2007). For example, Gimelli et al. (Gimelli et al. 2009) demonstrated the disruption of RNF139 in a young girl with dysgerminoma. Regarding the tumor-suppressive functions, Brauweiler et al. (Brauweiler et al. 2007) reported RNF139-caused inhibition of human kidney cell proliferation by inducing cell cycle arrest in G2/M phase, inhibiting DNA synthesis, and promoting apoptosis. Moreover, in a RING-dependent manner, RNF139 suppressed the tumor formation in a nude mouse model (Brauweiler et al. 2007). Lin et al. (2013) reported that RNF139 led to the ubiquitination and degradation of heme oxygenase-1 (HO-1), an antioxidant enzyme overexpressed in multiple cancers. In tongue cancer cells, RNF139 silencing dramatically enhanced cancer cell invasion and enhanced SCC25 cell tumorigenicity in nude mice (Wang et al. 2017). Considering the tumor-suppressive role of RNF139 in several cancers, we hypothesize that RNF139 also plays a tumor-suppressive role against glioma.

In the present study, firstly, we confirmed the mRNA expression and protein levels of RNF139 in tissue samples and cell lines. Secondly, RNF139 overexpression and knockdown was achieved in glioma cell lines and the specific effects of RNF139 overexpression or knockdown on cancer cell proliferation, apoptosis, migration, and invasion were investigated. Finally, considering that the occurrence of glioblastoma multiform, the most malignant glioma, is highly relevant with changes in the factors of the epidermal growth factor receptor (EGFR) and $\mathrm{PI} 3 \mathrm{~K} / \mathrm{Akt} /$ mechanistic target of rapamycin kinase (mTOR) signaling pathways (Chakravarti et al. 2004; Li et al. 2016), we investigated the alterations in the PI3K/AKT signaling in response to RNF139 overexpression or knockdown. Altogether, in the current study, for the first time, we tried to reveal the biological functions and the potential molecular mechanism of RNF139 in glioma. We observed that the tumor-suppressive role of RNF139 in glioma cells through regulating the possible downstream signaling (PI3K/AKT signaling). Our basic experimental outcomes provided sufficient results to uncover a newfound mRNA/signaling pathway regulatory network of RNF139/ $\mathrm{PI} 3 \mathrm{~K} / \mathrm{AKT}$ signaling in glioma, supplying a crucial perception concerning the regulatory theory in glioma advancement and new therapeutic options of glioma diseases.

\section{Materials and Methods}

\section{Clinical Samplin}

Twelve tissue samples from the tumor core region and twelve samples from the peritumoral brain edema (PTBE) region were obtained from patients received treatment (surgical resection) at Hunan Brain Hospital. The sampling procedure was conducted with the approval of the Ethic Committee of Hunan Brain Hospital. Immediately after specimen sampling, tissues were transferred to formalin or $-80{ }^{\circ} \mathrm{C}$ container until subsequent experiments.

\section{Cell Lineage and Cell Culture}

Normal human brain astroglia cell line SVG p12 (CRL8621) was obtained from ATCC (Manassas, VA, USA) and cultured in EMEM (Gibco, Waltham, MA, USA). Normal human astrocyte (HA; Catalog \#1800) was obtained from ScienCell (Carlsbad, CA, USA) and cultured in Astrocyte medium (AM; Catalog \#1801, ScienCell). Glioma cell line U87 (HTB-14) was obtained from ATCC and cultured in EMEM (Gibco). Glioma cell line SHG-44

Table 2 qRT-PCR primer sequences

\begin{tabular}{ll}
\hline Primer & Sequence $\left(5^{\prime}-3^{\prime}\right)$ \\
\hline RNF139 & \\
Forward primer & TACCCGGATTCCAGCCAAAG \\
Reverse primer & GGGACCTTTTCGAGGAAGCA \\
GAPDH & \\
Forward primer & AGCCACATCGCTCAGACAC \\
Reverse primer & GCCCAATACGACCAAATCC \\
\hline
\end{tabular}


(3131C0001000700048) was obtained from Cell Resource Center, Shanghai Institute of Life Sciences, Chinese Academy of Sciences (Shanghai, China) and cultured in RPMI 1640 (w/o Hepes; Gibco). Glioma cell line GOS-3 (ACC 408) was obtained from German Collection of Microorganisms and Cell Cultures (Braunschweig, Germany) and cultured in Dulbecco's MEM (Gibco). Glioma cell line TJ905
(3111C0001CCC000267) was obtained from Cell Resource Center, Shanghai Institute of Life Sciences, Chinese Academy of Sciences and cultured in DMEM-H: Dulbecco's modified Eagle's medium (DME H-21 4.5 g/Liter Glucose; Gibco). All glioma cell lines were cultured in medium supplemented with $10 \%$ FBS (Invitrogen, Waltham, MA, USA). All cells were cultured at $37^{\circ} \mathrm{C}$ in $5 \% \mathrm{CO}_{2}$.
A

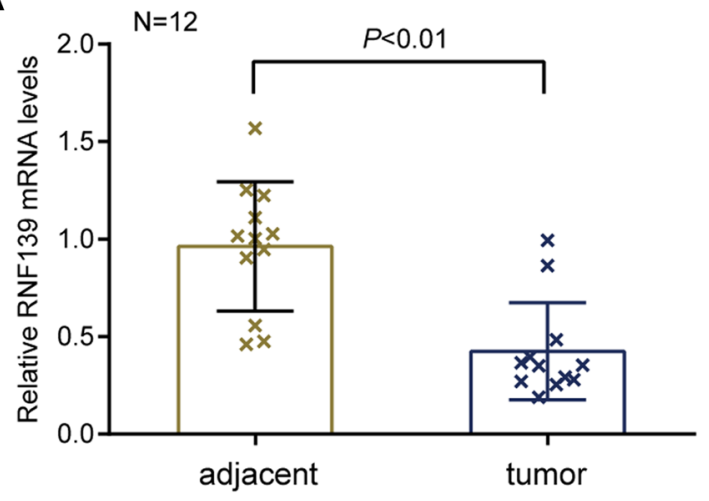

C

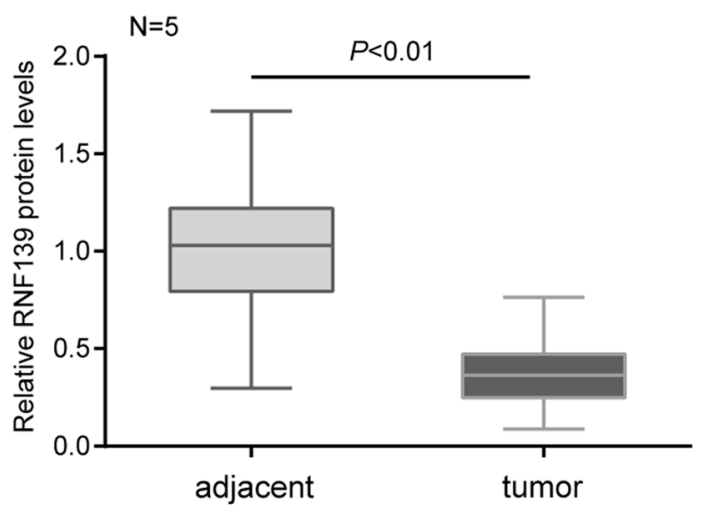

E

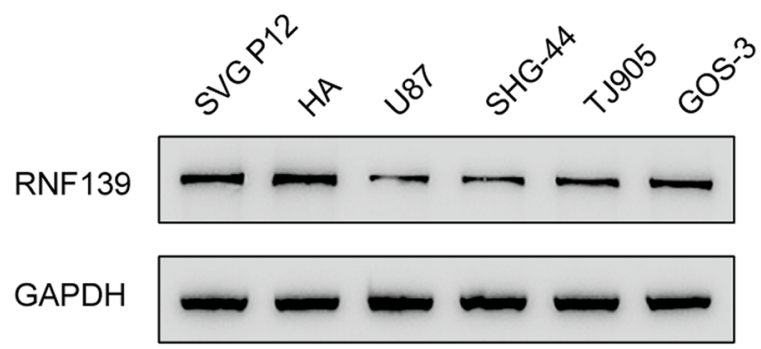

B

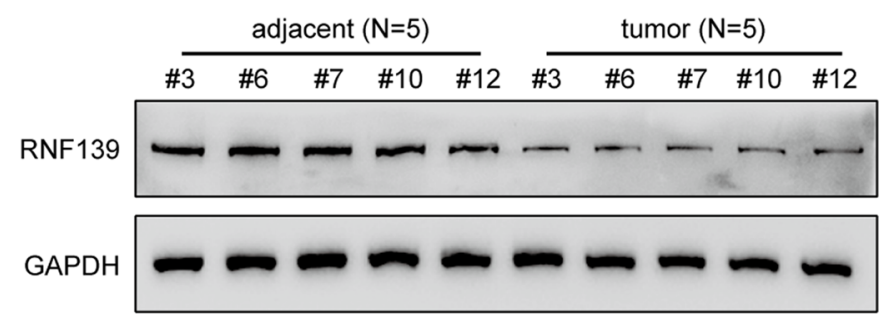

$\mathbf{D}$

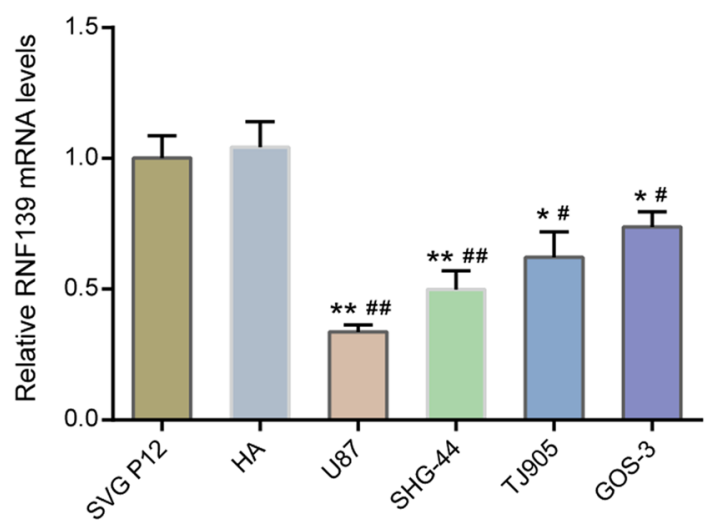

$\mathbf{F}$

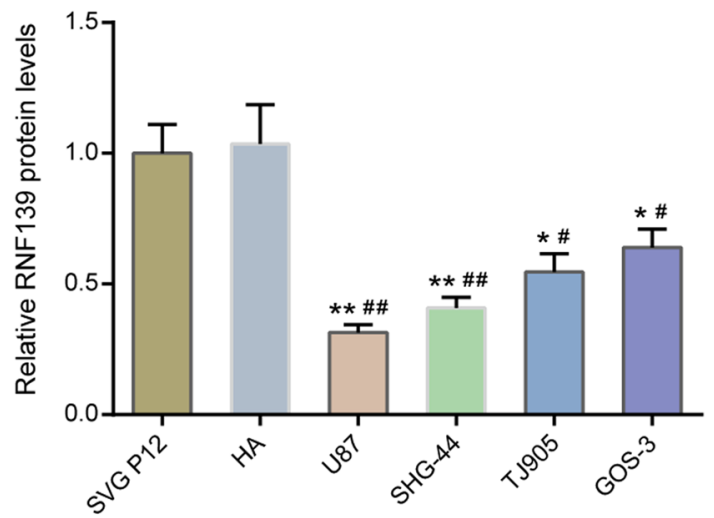

cell lines, U87, SHG-44, GOS-3, TJ905 by qRT-PCR. $N=3$. E, F The protein level of RNF139 was examined in human astrocyte cell lines, SVG P12 and HA, and glioma cell lines, U87, SHG-44, GOS-3, TJ905 by immunoblotting. $N=3, * P<0.05$, $* * P<0.01$ compared to SVG P12 group; $\# P<0.05, \# \# P<0.01$ compared to HA group 


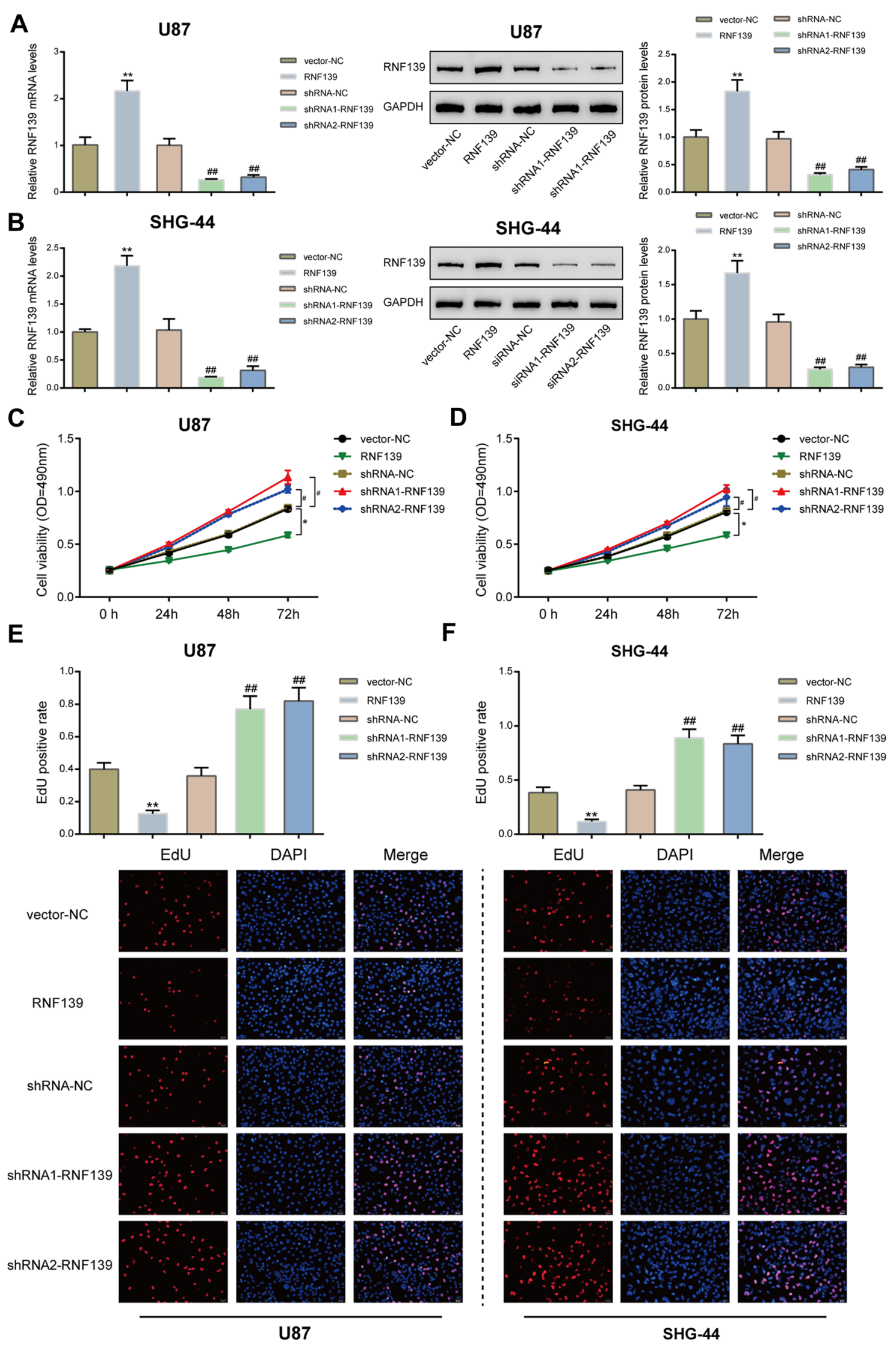


4Fig. 2 Effects of RNF139 overexpression and knockdown on glioma cell proliferation. A, B RNF139 overexpression or knockdown was achieved in U87 and SHG-44 cells by transfecting RNF139overexpressing vector (RNF139) or small interfering RNA targeting RNF139 (shRNA1-RNF139 and shRNA2-RNF139). The expression levels of RNF139 were confirmed by qRT-PCR and immunoblotting. Next, U87 and SHG-44 cells were transfected with RNF139 or shRNA1-RNF139/shRNA2-RNF139 and examined for cell viability by MTT assay $(\mathbf{C}, \mathbf{D})$ and DNA synthesis by EdU assay $(\mathbf{E}, \mathbf{F})$. $N=3, * P<0.05, * * P<0.01$ compared to vector-NC group; $\# P<0.05$, $\# \# P<0.01$ compared to shRNA-NC group

\section{Cell Transfection}

For RNF139 overexpression, full-length RNF139 gene was combined in pLVX-puro (RNF139) and the empty vector was used as a negative control (vector-NC). For RNF139 knockdown, short-hairpin RNA against RNF139 gene, as well as their nontargeting sequences were reconstructed in pLVX-shRNA2-puro (shRNA-NC, shRNA1-RNF139, shRNA2-RNF139; YouBio, China). The primer sequence for shRNA are listed in Table 1. Cells were seeded in a 24-well plate, and the Lipofectamine 3000 reagent (Thermo Fisher Scientific, Waltham, MA, USA) was used to transfect cells with the plasmids mentioned above. Cells were collected $48 \mathrm{~h}$ after transfection.

\section{qRT-PCR}

TRIzol reagent (Invitrogen) was used to extract total RNA from target cells or tissues, and then use the first strand cDNA synthesis kit (Promega) for reverse transcription. A SYBR Green PCR Master Mix (Qiagen) was used to detect the expression level according to the manufacturer's instructions. With GAPDH expression as an internal reference, calculate the relative expression using the $\mathrm{Ct}$ method. The specific primers used are presented in Table 2.

\section{Immunoblotting}

Extract total protein from target cells, separate the total protein samples using SDS-PAGE, and transfer the protein samples to polyvinylidene fluoride membranes. For the blockage of nonspecific binding, the membranes were incubated with $5 \%$ skim milk dissolved in diphenyltris buffer saline for $2 \mathrm{~h}$ at room temperature. Incubate the membranes with the primary antibodies against RNF139 (Catalog \# MBS421811; MyBioSource, Inc., San Diego, CA, USA), PI3K (AF6241; Affinity, Cincinnati, OH), p-PI3K (AF3242, Affinity), Akt (Y409094; ABM, Richmond, Canada), or p-Akt (Y011054, ABM) overnight at $4{ }^{\circ} \mathrm{C}$. The incubation with the primary antibodies was followed by another incubation with the corresponding horseradish peroxidase coupled secondary antibody for $2 \mathrm{~h}$ at room temperature. Finally, the enhanced chemiluminescence visualization scan was performed.

\section{MTT Assay for Cell Viability}

Target cells were transfected and seeded in 96-well plates at a density of $1 \times 10^{4}$ cells/well for a 24,48 , or $72 \mathrm{~h}$ of incubation. At the end of each incubation, $10 \mu \mathrm{l}$ of $5 \mathrm{mg} /$ ml MTT (added to PBS; Sigma-Aldrich, St. Louis, MO, USA) was added to each well and the cells were subjected to another $4 \mathrm{~h}$ of incubation. Remove the supernatants, add $100 \mu$ dimethyl sulfoxide (DMSO, Thermo Fisher Scientific, Waltham, MA, USA) to each well, measure the absorbance value at $490 \mathrm{~nm}$ using a microplate reader.

\section{EdU Assay for DNA Synthesis}

Use 5-ethyl-2'-deoxyuridine (EdU) detection kit (EdU kit; Ribobio, Guangzhou, China) for determining DNA synthesis. Cells were firstly transfected, incubated with EdU for $2 \mathrm{~h}$, and collected. Then, fix the cells in $4 \%$ formaldehyde for $20 \mathrm{~min}$ and permeabilize the cells. Wash the cells with PBS, treat the cells with $200 \mu$ A Apollo $®$ reaction cocktail (Ribobio) for $10 \mathrm{~min}$, and permeabilize the cells. Stain the cells with $100 \mu \mathrm{l}$ Hoechst 33,342 (5 $\mu \mathrm{g} / \mathrm{ml}$; Thermo Fisher Scientific). Observe the cells under a fluorescence microscope (Olympus, Tokyo, Japan).

\section{Flow Cytometry for Cell Apoptosis}

Target cells were transfected and examined for cell apoptosis using a FITC-labeled AnnexinV/propidium iodide (PI) Apoptosis Detection kit (Beyotime, Shanghai, China). Immediately after staining, flow cytometry was performed (Beckman, USA). Early apoptotic cells were positive for Annexin V, and late apoptotic cells were positive for both Annexin $\mathrm{V}$ and PI.

\section{Transwell for Cell Migration and Invasion}

Target cells were transfected and evaluated for the invasion using Matrigel Transwell (Corning BioCoat Matrigel Invasion Chambers; Corning, Corning, NY, USA). The cells were seeded in a 6 -well plate for $12 \mathrm{~h}$ at a density of $5 \times 10^{5}$ cells/well. Subsequently, the cells were cultured with serumfree medium for $12 \mathrm{~h}$ and then seeded in $100 \mu \mathrm{l}$ serum-free media into each well of the Transwell at a density of $2 \times 10^{4}$ cells/well. In the lower chamber, add $700 \mu$ l complete medium containing $10 \%$ FBS. After 48 h, remove the cells stayed on the upper chambers, fix the cells on the surface of the lower chamber with $4 \%$ paraformaldehyde, and stain these cells with crystal violet. Count the number of cells on the surface of the lower chamber (invaded cells). Cell 
A
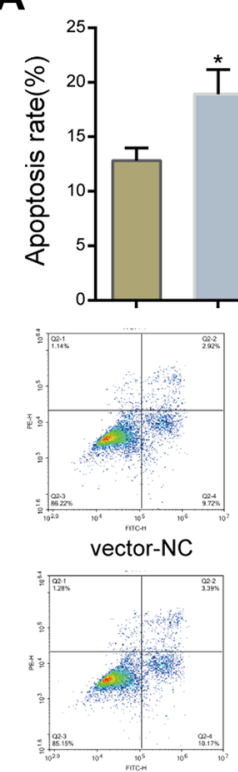

ShRNA-NC

\section{C}
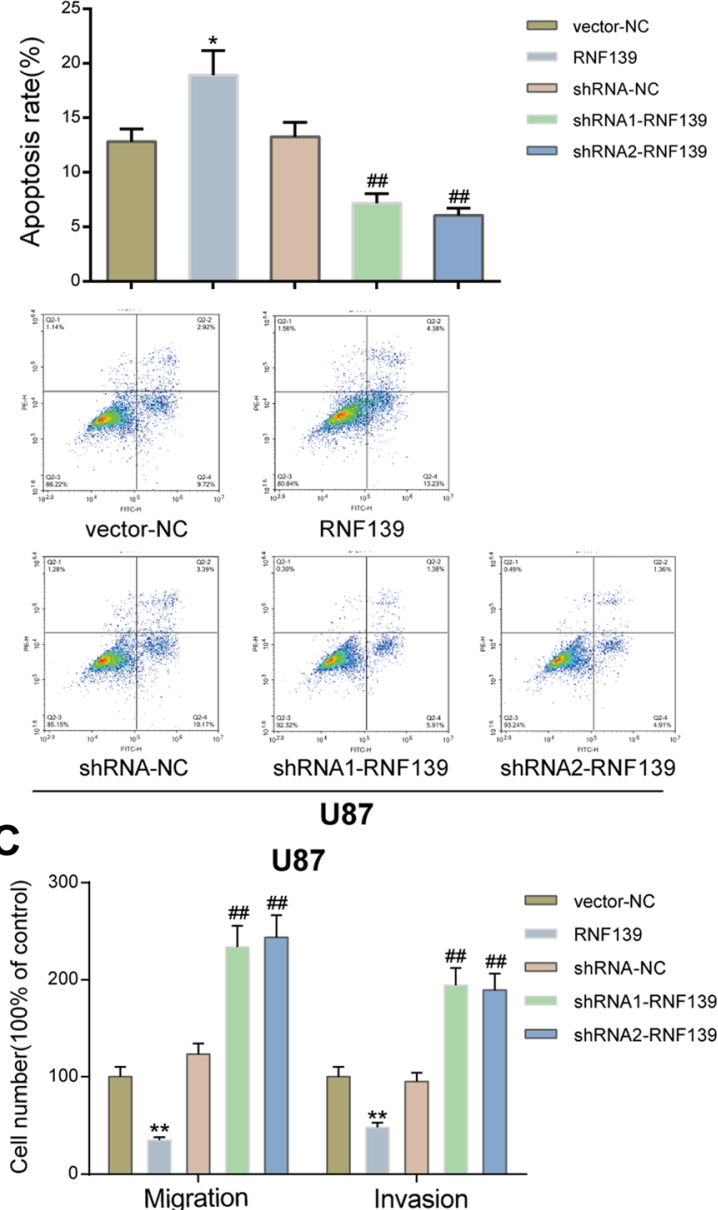

ShRNA1-RNF139
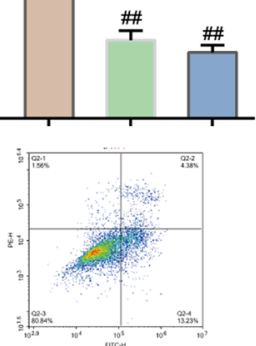

RNF139

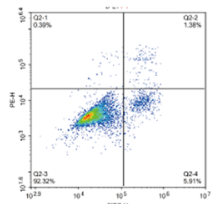

ShRNA1-RNF139

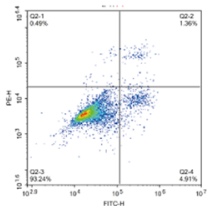

ShRNA2-RNF139

B

\section{D}
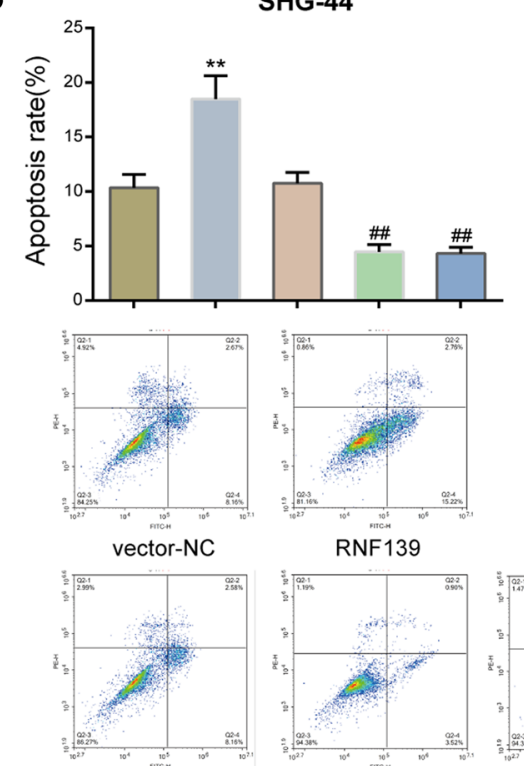

vector-NC

- RNF139

$\square$ ShRNA-NC

- ShRNA1-RNF139

\ShRNA2-RNF139
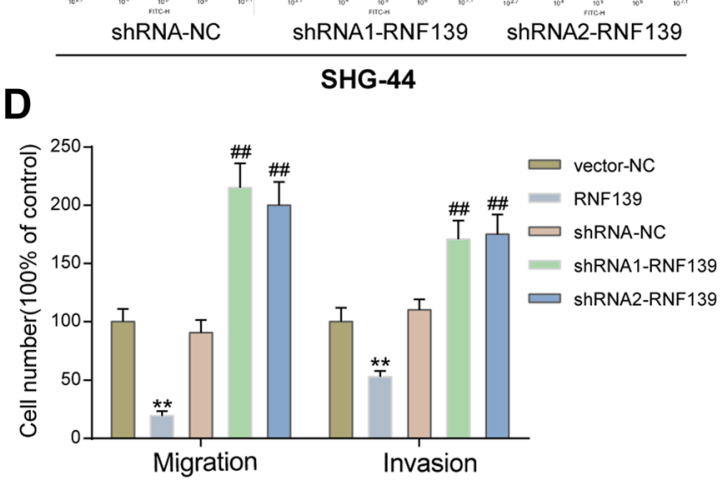

E

RNF139

shRNA-NC

ShRNA1-RNF139 ShRNA2-RNF139

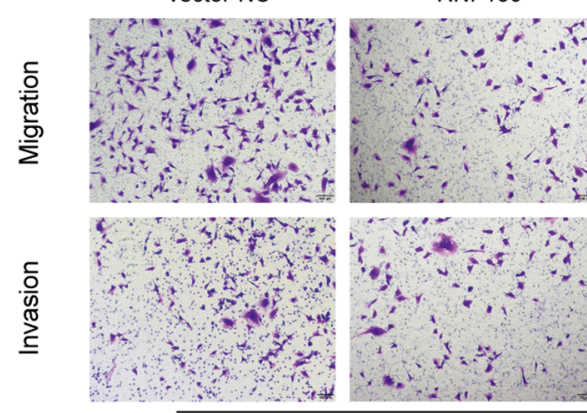

$\mathbf{F}$

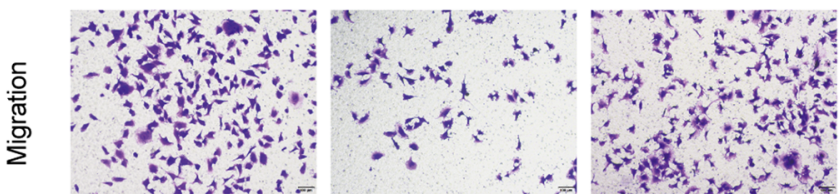

U87

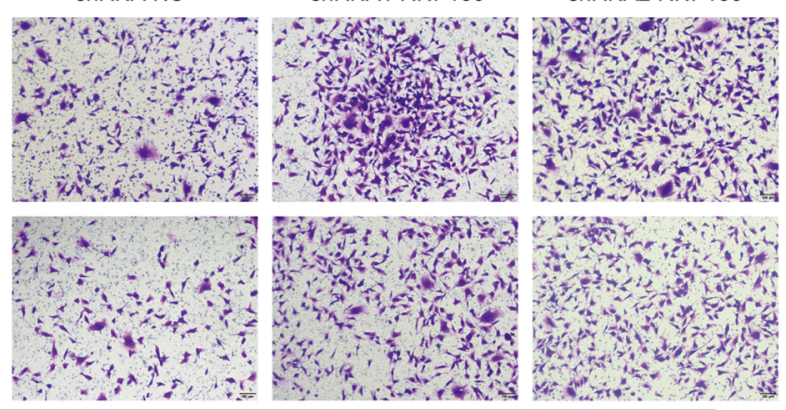

at:
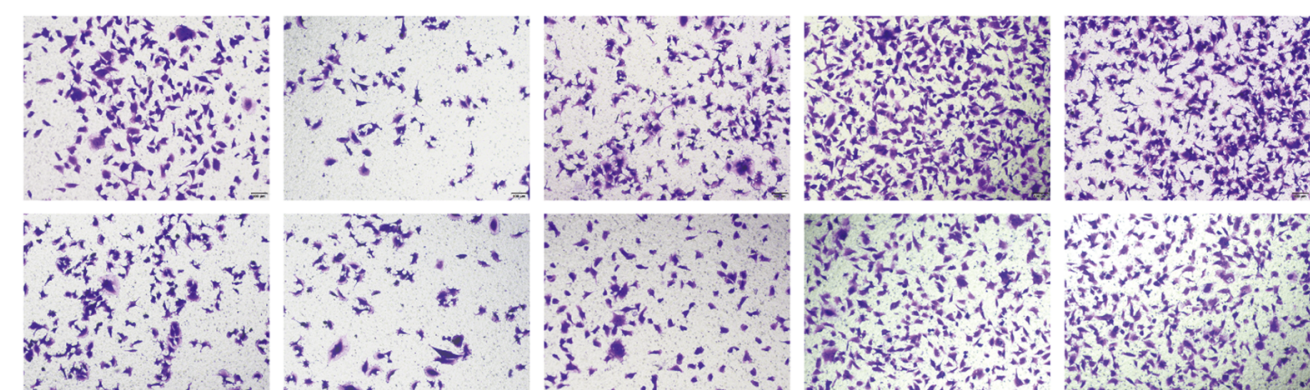

SHG-44 
4Fig. 3 Effects of RNF139 overexpression and knockdown on glioma cell apoptosis, migration, and invasion. U87 and SHG-44 cells were transfected with RNF139 or shRNA1-RNF139/shRNA2-RNF139 and examined for cell apoptosis by flow cytometry $(\mathbf{A}, \mathbf{B})$ and cell migration and invasion by Transwell assay $(\mathbf{C}-\mathbf{F}) . N=3, * P<0.05$ compared to vector-NC group; $* * P<0.01$, \#\#P<0.01 compared to shRNA-NC group

migration was evaluated similarly except for using Transwell without Matrigel.

\section{Statistics Analysis}

Each experiment was repeated for at least three times. Data were processed using the GraphPad software (San Diego, CA, USA) and shown as mean \pm standard deviation (SD). A one-way analysis of variance (ANOVA) and then Tukey's multiple comparison test or Student's $t$ test was performed to analyze the statistical significance. The cell experiments were performed at least three times. A $P$ value of $<0.05$ was considered statistically significant.

\section{Results}

\section{The Downregulation of RNF139 in Glioma Tissues and Cell Lines}

Before investigating the specific effects of RNF139 on glioma cell phenotypes, we firstly verified tissue RNF139 expression in glioma and adjacent noncancerous specimens; as shown in Fig. 1A, RNF139 mRNA levels were remarkably downregulated in tumor tissues compared with the control group $(N=12)$. Consistently, the protein levels of RNF139 were significantly decreased in tumor tissues compared with
A

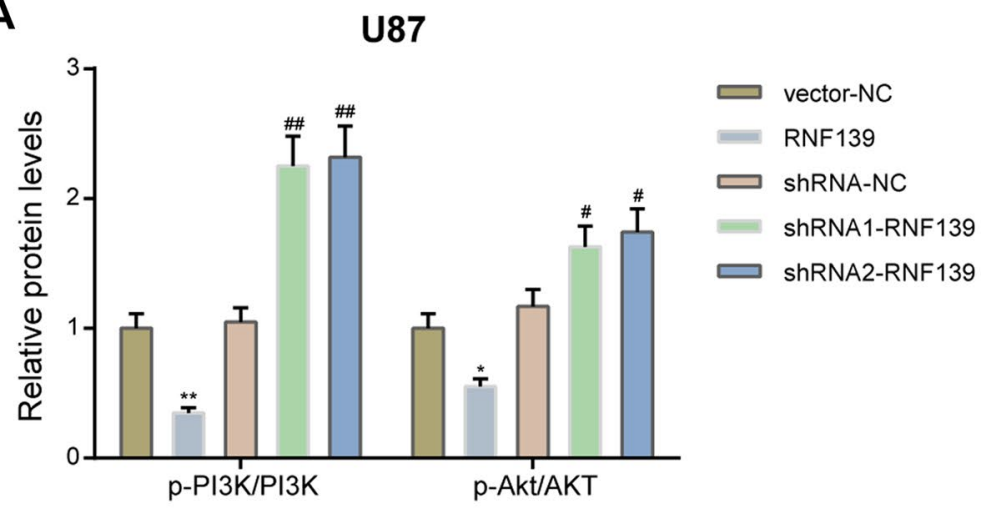

B

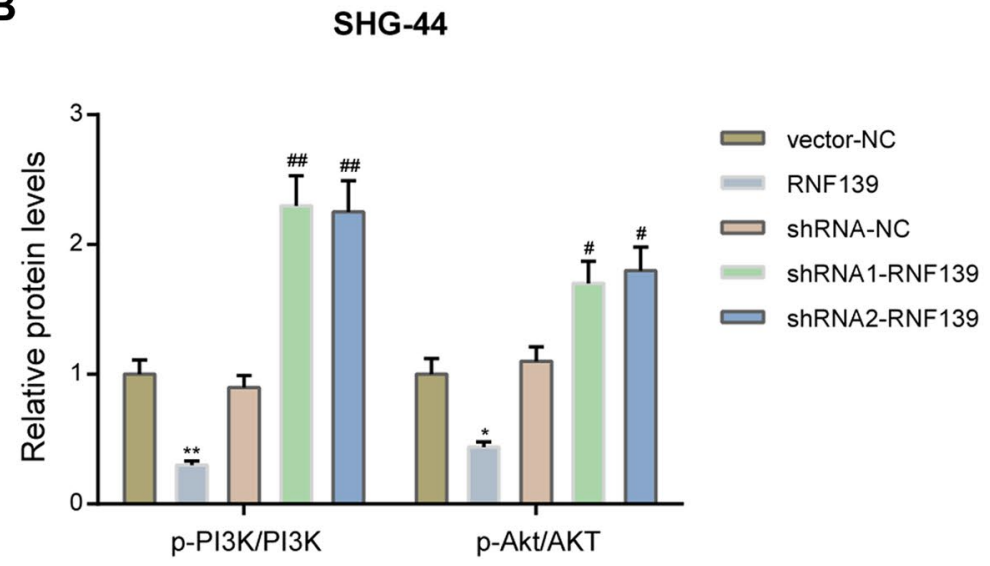

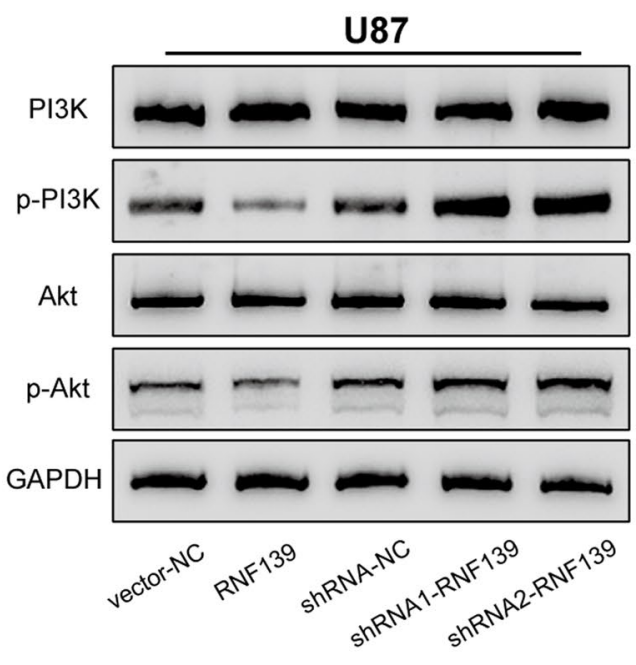

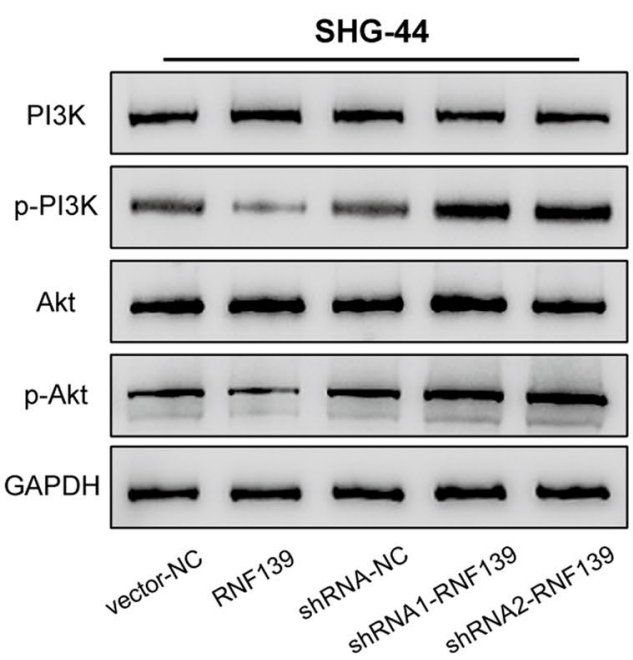

Fig. 4 The PI3K/AKT signaling pathway is involved in the effects of RNF139. U87 and SHG-44 cells were transfected with RNF139 or shRNA1-RNF139/shRNA2-RNF139 and examined for the protein levels of PI3K, p-PI3K, Akt, and p-Akt by immunoblotting $(\mathbf{A}, \mathbf{B})$. $N=3, * P<0.05, * * P<0.01$ compared to vector-NC group; $\# P<0.05$, $\# \# P<0.01$ compared to shRNA-NC group 
those in adjacent non-cancerous tissues $(N=5)$ (Fig. 1B, C). In cell lines, the expression of RNF139 was dramatically downregulated in glioma cell lines, including U87, SHG-44, GOS-3, and TJ905 compared with that in human astrocyte cell lines, SVG P12 and HA (Fig. 1D). Similarly, the protein levels of RNF139 were significantly decreased in glioma cell lines, including U87, SHG-44, GOS-3, and TJ905 compared with those in human astrocyte cell lines, SVG P12 and HA (Fig. 1E, F). These findings confirm the abnormal downregulation of RNF135 in glioma.

\section{Effects of RNF139 Overexpression and Knockdown on Glioma Cell Phenotypes}

The abnormal downregulation of RNF139 suggests that it may serve as a tumor-suppressor against glioma. For verifying the speculation, we achieved RNF139 overexpression or knockdown in U87 and SHG-44 cells by transfecting RNF139-overexpressing vector (RNF139) or small interfering RNA targeting RNF139 (shRNA1-RNF139 and shRNA2-RNF139). The mRNA expression and protein levels of RNF139 were confirmed by qRT-PCR and Immunoblotting (Fig. 2A, B); RNF139 overexpression or knockdown was successfully conducted. Next, U87 and SHG-44 cells were transfected with RNF139 or shRNA1-RNF139/ shRNA2-RNF139 and examined for cell viability, DNA synthesis, cell apoptosis, cell migration, and cell invasion. In both glioma cell lines, RNF139 overexpression significantly inhibited tumor cell viability (Fig. 2C, D), DNA synthesis (Fig. 2E, F), and cell migration and invasion (Fig. 3C-F), whereas promoted cell apoptosis (Fig. 3A, B). By contrast, when RNF139 was knocked down in glioma cell lines, cell viability (Fig. 2C, D), DNA synthesis (Fig. 2E, F), and cell migration and invasion (Fig. 3C-F) were all enhanced, whereas cell apoptosis was inhibited (Fig. 3A, B). These data indicate that ectopic RNF139 overexpression could inhibit, whereas RNF139 knockdown could aggravate the aggressive behaviors of glioma cells.

\section{RNF139 Could Affect the Activation of the PI3K/AKT Signaling Pathway}

The aggressiveness of cancer cells could result from the deregulation of complex alternate signaling pathways (Alifieris and Trafalis 2015); among the deregulated signaling pathways, the PI3K/AKT signaling showed to be a crucial one in glioma pathogenesis (Tong et al. 2016; Wen and Kesari 2008; Zhao et al. 2017). Therefore, we investigated whether the PI3K/AKT signaling pathway could also be affected by RNF139 in glioma cells. U87 and SHG-44 cells were transfected with RNF139 or shRNA1-RNF139/ shRNA2-RNF139 and examined for the protein levels of PI3K, p-PI3K, Akt, and p-Akt. As shown in Fig. 4A, B, in both glioma cell lines, RNF139 overexpression dramatically decreased the ratio of p-PI3K/PI3K and p-AKT/AKT; on the contrary, RNF139 knockdown induced by either shRNA1RNF139 or shRNA2-RNF139 elevated the ratio of p-PI3K/ PI3K and p-AKT/AKT. Thus, RNF139 overexpression could inhibit the hyper-activation of the PI3K/AKT signaling pathway in glioma cells.

\section{Discussion}

In the present study, we firstly confirmed the abnormal downregulation of RNF139 in glioma tissues and cell lines. In glioma cells, ectopic RNF139 overexpression could inhibit, whereas RNF139 knockdown could aggravate the aggressive behaviors of glioma cells, including hyperproliferation, migration, and invasion. Moreover, in two glioma cell lines, for the first time, RNF139 was identified that played a tumor suppressor role through regulating PI3K/ AKT signaling. RNF139 overexpression inhibited, whereas RNF139 knockdown enhanced the phosphorylation of PI3K and AKT.

Similar as phosphorylation, ubiquitination is another most prevalent and critical protein modifications occurring inside the cell. Since ubiquitination could occur in a wide range of proteins, ubiquitination participates in cellular processes including cell cycle progression, cell apoptosis, receptor downregulation, and gene transcription. Thus, not surprisingly, ubiquitination has been described in multiple cancer-related pathways. During ubiquitination, a ubiquitin is connected to the target protein by a covalent isopeptide bond between ubiquitin C-terminal and target protein lysine residue. Because of its role in interacting with substrates and determining the substrate specificity, the E3 ubiquitin ligases are crucial in the ubiquitinconjugation system. Not surprisingly, the functions of E3 ligases have been reported in cancers, either by dictating the degradation of oncogenic proteins or tumor-suppressive proteins. As we have mentioned, FBW7 agonist leads to the degradation of c-MYC and cyclin $\mathrm{E}$ in cancer cells (Diefenbacher et al. 2015). Another group demonstrated that nuclear TRIM59, as an E3 ligase, led to the ubiquitination of mH2A1 and affected mH2A1 stability in glioblastoma (Sang et al. 2019). Similarly, stable SHPRH, as an E3 ligase, ubiquitinated proliferating cell nuclear antigen (PCNA), thus inhibiting glioma cell proliferation and tumorigenicity (Zhang et al. 2018). On the contrary, the ubiquitin-protein ligase E3C (UBE3C) led to the ubiquitination and degrading of Annexin A7, promoting glioma development (Pan et al. 2015). In the present study, we firstly found the abnormal downregulation of RNF139, an E3 ubiquitin ligase, in glioma tissue samples and cell lines. 
The downregulation of RNF139 in glioma suggests that RNF139 might play a role in glioma development.

Interestingly, RNF139 has been previously regarded as a tumor-suppressive factor in several cancers, including tongue cancer (Wang et al. 2017), renal cancer (Brauweiler et al. 2007), kidney carcinoma A498 cells, osteosarcoma U2OS cells, and cervical carcinoma HeLa cells (Lin et al. 2013). In these cancer cells, ectopic RNF139 expression has been reported to induce cell cycle arrest, suppress cancer cell proliferation, migration and invasion, and inhibit the tumor formation in nude mice. Glioma is characterized by rapid cell proliferation and angiogenesis (Peng et al. 2018). Besides, similar to the spreading process of metastasis, single glioma cells could be observed in the far side of the brain (Kirsch et al. 2000). Thus, to investigate the specific role of RNF139 in glioma carcinogenesis, we conducted ectopic expression or knockdown of RNF139 in glioma cells and examined the cell viability, DNA synthesis, cell apoptosis, migration, and invasion. Consistent with its abnormal downregulation in glioma, ectopic RNF139 expression significantly suppressed glioma cell proliferation, migration and invasion, and promoted cell apoptosis; RNF139 knockdown exerted opposite effects on glioma cell phenotypes. These findings strongly support the tumor-suppressive role of RNF139 in glioma.

Genetic aberrations have been frequently found in glioblastoma, one of the most malignant gliomas. These aberrations include EGFR, platelet-derived growth factor receptor alpha (PDGFRA), phosphatidylinositol-4,5-bisphosphate 3-kinase catalytic subunit alpha (PIK3CA), phosphatase and tensin homolog (PTEN), tumor protein p53 (TP53), cyclin-dependent kinase inhibitor 2A/B (CDKN2A/B), and so on, leading to dysfunction of the PI3K/Akt/mTOR, p53, RB transcriptional corepressor 1 (RB1), and other signaling pathways; however, the discovery of these aberrations also open up targeted therapies through targeting hyperactivated pathways [4]. The crucial role of the PI3K/Akt signaling pathway has been widely recognized in mediating cell proliferation, apoptosis, motility, as well as angiogenesis in glioblastoma (Brennan et al. 2013). Due to the cellular functions, elevated PI3K and AKT are poor prognostic factor in patients with malignant gliomas (Chakravarti et al. 2004). In the present study, we also monitored the alterations in the $\mathrm{PI} 3 \mathrm{~K} / \mathrm{AKT}$ signaling in response to RNF139 overexpression or knockdown. Consistent with previous studies, RNF139 overexpression inhibited, whereas RNF139 knockdown enhanced the phosphorylation of PI3K and AKT. These findings suggest that the PI3K/AKT signaling might mediate the tumor-suppressive role of RNF139 in glioma for the first time.

Altogether, we demonstrate the aberration in RNF139 expression in glioma tissue samples and cell lines. RNF139 serves as a tumor-suppressor in glioma by inhibiting cancer cell proliferation, migration, and invasion and promoting cancer cell apoptosis through regulating PI3K/AKT signaling.

Abbreviations AKT: AKT serine/threonine kinase 1; CDKN2A/B: Cyclin-dependent kinase inhibitor 2A/B; EdU: 5-Ethyl2'-deoxyuridine; EGFR: Epidermal growth factor receptor; HO1: Heme oxygenase-1; mTOR: Mechanistic target of rapamycin kinase; PCNA: Proliferating cell nuclear antigen; PDGFRA: Platelet-derived growth factor receptor alpha; PI: Propidium iodide; PIK3CA: Phosphatidylinositol-4,5-bisphosphate 3-kinase catalytic subunit alpha; PI3K: Phosphatidylinositol 3-kinase; PTBE: Peritumoral brain edema; PTEN: Phosphatase and tensin homolog; RB1: RB transcriptional corepressor 1; RNF139: Ring finger protein 139; TP53: Tumor protein $\mathrm{p} 53$; UBE3C: Ubiquitin-protein ligase E3C

Author Contribution Xiaofeng Chen, Liang Li, and Shucheng Zou made substantial contribution to the conception and design of the work; Weiping Kuang, Yong Zhu, and Bin Zhou analyzed and interpreted the data; Xiaofeng Chen, Xiaosong Li, and Xi Zhang drafted the manuscript; Bo Li and Liang Li revised the work critically for important intellectual content; Liang Li and Shucheng Zou collected grants; final approval of the work: all authors.

Funding This study was supported by Hunan Science and technology major special program (2018SK1030), Natural Science Foundation of Hunan Province (2019JJ80024 and 2020JJ8081), Hunan Provincial Health and Family Planning Commission Project (C2019043), and Hunan Province Science and Technology Innovation Plan Project (2018SK50605).

Availability of Data and Materials Please contact the authors for data requests.

\section{Declarations}

Ethics Approval and Consent to Participate All procedures performed in studies involving human participants were in accordance with the ethical standards of Hunan Brain Hospital and with the 1964 Helsinki declaration. Written informed consent was obtained from all patients.

Consent for Publication Consent for publication was obtained from the participants.

Competing Interests The authors declare no competing interests.

Open Access This article is licensed under a Creative Commons Attribution 4.0 International License, which permits use, sharing, adaptation, distribution and reproduction in any medium or format, as long as you give appropriate credit to the original author(s) and the source, provide a link to the Creative Commons licence, and indicate if changes were made. The images or other third party material in this article are included in the article's Creative Commons licence, unless indicated otherwise in a credit line to the material. If material is not included in the article's Creative Commons licence and your intended use is not permitted by statutory regulation or exceeds the permitted use, you will need to obtain permission directly from the copyright holder. To view a copy of this licence, visit http://creativecommons.org/licenses/by/4.0/. 


\section{References}

Alifieris C, Trafalis DT (2015) Glioblastoma multiforme: Pathogenesis and treatment. Pharmacol Ther 152:63-82. https://doi.org/10. 1016/j.pharmthera.2015.05.005

Brauweiler A et al (2007) RING-dependent tumor suppression and G2/M arrest induced by the TRC 8 hereditary kidney cancer gene. Oncogene 26:2263-2271. https://doi.org/10.1038/sj.onc.1210017

Brennan CW et al (2013) The Somatic Genomic Landscape of Glioblastoma Cell 155:462-477. https://doi.org/10.1016/j.cell.2013. 09.034

Chakravarti A, Zhai G, Suzuki Y, Sarkesh S, Black PM, Muzikansky A, Loeffler JS (2004) The prognostic significance of phosphatidylinositol 3-kinase pathway activation in human gliomas. J Clin Oncol 22:1926-1933. https://doi.org/10.1200/JCO.2004.07.193

Chen Y, Hu F, Zhou Y, Chen W, Shao H, Zhang Y (2013) MGMT promoter methylation and glioblastoma prognosis: a systematic review and meta-analysis. Arch Med Res 44:281-290. https://doi. org/10.1016/j.arcmed.2013.04.004

Ciechanover A (2005) Proteolysis: From the lysosome to ubiquitin and the proteasome. Nat Rev Mol Cell Biol 6:79-87. https://doi.org/ $10.1038 / \mathrm{nrm} 1552$

Ciechanover A, DiGiuseppe JA, Bercovich B, Orian A, Richter JD, Schwartz AL, Brodeur GM (1991) Degradation of nuclear oncoproteins by the ubiquitin system in vitro. Proc Natl Acad Sci U S A 88:139-143. https://doi.org/10.1073/pnas.88.1.139

Ciechanover A, Finley D, Varshavsky A (1984) Ubiquitin dependence of selective protein degradation demonstrated in the mammalian cell cycle mutant ts85. Cell 37:57-66. https://doi.org/10.1016/ 0092-8674(84)90300-3

Diefenbacher ME, Chakraborty A, Blake SM, Mitter R, Popov N, Eilers M, Behrens A (2015) Usp28 counteracts Fbw7 in intestinal homeostasis and cancer. Cancer Res 75:1181-1186. https://doi.org/10. 1158/0008-5472.CAN-14-1726

Esteller $M$ et al (2000) Inactivation of the DNA-repair gene MGMT and the clinical response of gliomas to alkylating agents. N Engl J Med 343:1350-1354. https://doi.org/10.1056/ NEJM200011093431901

Gemmill RM et al (1998) The hereditary renal cell carcinoma 3;8 translocation fuses FHIT to a patched-related gene, TRC8. Proc Natl Acad Sci U S A 95:9572-9577. https://doi.org/10.1073/ pnas.95.16.9572

Ghosh S, May MJ, Kopp EB (1998) NF-kappa B and Rel proteins: evolutionarily conserved mediators of immune responses. Annu Rev Immunol 16:225-260. https://doi.org/10.1146/annurev. immunol.16.1.225

Gimelli S et al (2009) The tumor suppressor gene TRC8/RNF139 is disrupted by a constitutional balanced translocation $\mathrm{t}(8 ; 22)$ (q24.13;q11.21) in a young girl with dysgerminoma. Mol Cancer 8:52 https://doi.org/10.1186/1476-4598-8-52

Kibel A, Iliopoulos O, DeCaprio JA, Kaelin WG Jr (1995) Binding of the von Hippel-Lindau tumor suppressor protein to elongin B and C. Science 269:1444-1446. https://doi.org/10.1126/scien ce. 7660130

Kirsch M, Schackert G, Black PM (2000) Angiogenesis, metastasis, and endogenous inhibition. J Neurooncol 50:173-180. https:// doi.org/10.1023/a:1006453428013

Koepp DM, Harper JW, Elledge SJ (1999) How the cyclin became a cyclin: regulated proteolysis in the cell cycle. Cell 97:431-434. https://doi.org/10.1016/s0092-8674(00)80753-9
Li X et al (2016) PI3K/Akt/mTOR signaling pathway and targeted therapy for glioblastoma Oncotarget 7:33440-33450 https://doi. org/10.18632/oncotarget.7961

Lin PH, Lan WM, Chau LY (2013) TRC8 suppresses tumorigenesis through targeting heme oxygenase- 1 for ubiquitination and degradation. Oncogene 32:2325-2334. https://doi.org/10.1038/ onc. 2012.244

Muratani M, Tansey WP (2003) How the ubiquitin-proteasome system controls transcription. Nat Rev Mol Cell Biol 4:192-201. https://doi.org/10.1038/nrm1049

Ostrom QT et al (2014) The epidemiology of glioma in adults: a "state of the science" review. Neuro Oncol 16:896-913. https:// doi.org/10.1093/neuonc/nou087

Pan SJ et al (2015) Ubiquitin-protein ligase E3C promotes glioma progression by mediating the ubiquitination and degrading of annexin A7. Sci Rep 5:11066. https://doi.org/10.1038/ srep 11066

Peng Z, Liu C, Wu M (2018) New insights into long noncoding RNAs and their roles in glioma. Mol Cancer 17:61. https://doi. org/10.1186/s12943-018-0812-2

Pickart CM (2001) Mechanisms underlying ubiquitination. Annu Rev Biochem 70:503-533. https://doi.org/10.1146/annurev.biochem. 70.1.503

Rock KL, Goldberg AL (1999) Degradation of cell proteins and the generation of MHC class I-presented peptides. Annu Rev Immunol 17:739-779. https://doi.org/10.1146/annurev.immunol.17.1. 739

Rogers S, Wells R, Rechsteiner M (1986) Amino acid sequences common to rapidly degraded proteins: the PEST hypothesis. Science 234:364-368. https://doi.org/10.1126/science.2876518

Sang Y et al (2019) CDK5-dependent phosphorylation and nuclear translocation of TRIM59 promotes macroH2A1 ubiquitination and tumorigenicity. Nat Commun 10:4013. https://doi.org/10. 1038/s41467-019-12001-2

Tong Q et al (2016) LincRNA-Cox2 modulates TNF-alpha-induced transcription of Il12b gene in intestinal epithelial cells through regulation of $\mathrm{Mi}-2 / \mathrm{NuRD}-$ mediated epigenetic histone modifications. FASEB J 30:1187-1197. https://doi.org/10.1096/fj. $15-279166$

Wang L, Yin W, Shi C (2017) E3 ubiquitin ligase, RNF139, inhibits the progression of tongue cancer. BMC Cancer 17:452. https:// doi.org/10.1186/s12885-017-3438-7

Wen PY, Kesari S (2008) Malignant gliomas in adults. N Engl J Med 359:492-507. https://doi.org/10.1056/NEJMra0708126

Zhang $\mathrm{M}$ et al (2018) A novel protein encoded by the circular form of the SHPRH gene suppresses glioma tumorigenesis. Oncogene 37:1805-1814. https://doi.org/10.1038/s41388-017-0019-9

Zhao HF, Wang J, Shao W, Wu CP, Chen ZP, To ST, Li WP (2017) Recent advances in the use of PI3K inhibitors for glioblastoma multiforme: current preclinical and clinical development. Mol Cancer 16:100. https://doi.org/10.1186/s12943-017-0670-3

Zheng N, Shabek N (2017) Ubiquitin ligases: structure, function, and regulation. Annu Rev Biochem 86:129-157. https://doi.org/10. 1146/annurev-biochem-060815-014922

Publisher's Note Springer Nature remains neutral with regard to jurisdictional claims in published maps and institutional affiliations. 